\title{
[9] The Six-Jump Cycle Diffusion Mechanism in Non-Stoichiometric B2 Intermetallics: the Vacancy-Wind Factor
}

\author{
I.V.Belova and G.E.Murch \\ Diffusion in Solids Group, School of Engineering, \\ University of Newcastle, Callaghan, NSW 2308, \\ Australia
}

Keywords: 6-Jump Cycle, B2 Intermetallics, Vacancy-Wind

\begin{abstract}
In this paper we explore the behaviour of the vacancy-wind factor or Manning factor for chemical diffusion in antistructurally disordered intermetallics taking the B2 structure. The analytical formalism is based on generalized six-jump- cycles providing the vehicles for diffusion of both components. Comparison of the analytical results is made with results from Monte Carlo calculations based only on single vacancy jumps. Very good agreement is found at low temperatures/ high levels of order where it can be anticipated that six-jump-cycles dominate the diffusion process. In such circumstances, as a first (rough) approximation, it would be reasonable to put the vacancy-wind factor/Manning factor equal to 0.5 rather than unity as is often done.
\end{abstract}

\section{Introduction}

Discovered by John Manning in the early 1960s the vacancy-wind effect (in its chemical diffusion manifestation) is a subtle diffusion correlation effect arising in binary and higher order alloys whenever a flux of vacancies is directed preferentially towards atoms of one species. Manning [1, 2] pioneered the first calculations describing the vacancy-wind effect in binary concentrated (random) alloys where it results in an additional factor, $\mathrm{S}$, in the Darken equation linking the interdiffusion coefficient, $\mathrm{D}$, the tracer diffusion coefficients, $\mathrm{D}_{\mathrm{A}^{*}}$ and $\mathrm{D}_{\mathrm{B}^{*}}$, and the thermodynamic factor, $\phi$ :

$$
\widetilde{\mathrm{D}}=\phi \mathrm{S}\left(\mathrm{c}_{\mathrm{A}} \mathrm{D}_{\mathrm{B}^{*}}+\mathrm{c}_{\mathrm{B}} \mathrm{D}_{\mathrm{A}^{*}}\right)
$$

where $\mathrm{S}$ was given approximately by Manning as $[1,2]$ :

$$
\mathrm{S}=1+\frac{1-\mathrm{f}_{0}}{\mathrm{f}_{0}} \frac{\mathrm{c}_{\mathrm{A}} \mathrm{c}_{\mathrm{B}}\left(\mathrm{D}_{\mathrm{A}^{*}}-\mathrm{D}_{\mathrm{B}^{*}}\right)^{2}}{\left(\mathrm{c}_{\mathrm{A}} \mathrm{D}_{\mathrm{A}^{*}}+\mathrm{c}_{\mathrm{B}} \mathrm{D}_{\mathrm{B}^{*}}\right)\left(\mathrm{c}_{\mathrm{A}} \mathrm{D}_{\mathrm{B}^{*}}+\mathrm{c}_{\mathrm{B}} \mathrm{D}_{\mathrm{A}^{*}}\right)},
$$


where $f_{0}$ is the geometric tracer correlation factor for the structure, e.g. $f_{0}=0.7272$ in the bec lattice and $\mathrm{c}_{\mathrm{A}(\mathrm{B})}$ are the compositions of $\mathrm{A}(\mathrm{B})$ atoms. Eqn 2 can be readily reexpressed in terms of the tracer correlation factors:

$$
S=1+\frac{1-f_{0}}{f_{0}} \frac{c_{A} c_{B}\left(g f_{B}-f_{A}\right)^{2}}{\left(c_{A} f_{A}+c_{B} g f_{B}\right)\left(c_{A} g f_{B}+c_{B} f_{A}\right)}
$$

where $f_{A}$ and $f_{B}$ are the tracer correlation factors of the $A$ and $B$ atoms and $g$ is the ratio of the number of $A$ jumps per atom to the number of $B$ jumps per atom.

The vacancy-wind factor, $\mathrm{S}$, also called the Manning factor, has the limits $1.0<\mathrm{S}<$ $\mathrm{f}_{0}^{-1}$ in the Manning formalism. Eqn 2 has been very widely used, not just in disordered alloys that can be reasonably well approximated by the concentrated random model, but also in intermetallic compounds. In the latter materials, the use of Manning's expression was largely driven by the fact that there is simply nothing else available. Sometimes $\mathrm{S}$ is simply approximated by unity. Given the considerable uncertainties in the values of the various quantities in Eqn 1, especially the thermodynamic factor, this would appear to be reasonable as a first approximation.

Some years ago, the present authors [3] showed that Manning's original arguments for the random alloy could be extended to an analogous model in equi-atomic intermetallic compounds with the B1 or B2 structures showing antistructural disorder and intersublattice jumps. In this case, Eqns 2 and 3 for $\mathrm{S}$ still result. Various computer simulations have also verified the essential correctness of Eqns $2 / 3$, not only in the equiatomic intermetallics, but also in intermetallics taking the $\mathrm{Al} 15, \mathrm{Ll}_{2}$ and $\mathrm{DO}_{3}$ structures [46]. (In the A15 structure the meaning of $f_{0}$ needed to be extended somewhat to describe the structure actually explored.) All of these simulations were performed at fairly low levels of order.

It has been known for many years that at low temperatures or in highly ordered configurations vacancies cannot possibly move randomly because of the large amount of antistructural disorder (of high energy) that would result. At the stoichiometric composition it has been shown that a special six-jump-cycle (6JC) mechanism operates $[7,8]$. This is the lowest energy penalty jump sequence commensurate with effective diffusion. It is shown in Figure 1 in two-dimensional form. Starting from a perfectly ordered environment the vacancy progressively disorders the lattice in its first three jumps by putting three atoms onto their 'wrong' sublattices. In its subsequent three jumps the 
vacancy progressively re-orders the lattice by putting such atoms back onto their home sublattices (but in new positions). In the process there have been net displacements of three atoms: one atom ( $\mathrm{A}$ in the figure) has, in effect, exchanged with the vacancy across the diagonal of the cycle. The remaining two atoms $\left(B_{1}\right.$ and $B_{2}$ in the figure) have, in effect, exchanged positions across the other diagonal of the cycle. It should be noted that the vacancy will make many jump reversals on its way around the cycle as atoms jump to the wrong sublattice but retrace that jump a very short time later. Computer simulations of various alloy models describing antistructurally disordered B1 and B2 alloys have clearly shown the emergence of the $6 \mathrm{JC}$ mechanism as the degree of order increases $[9$, $10]$.
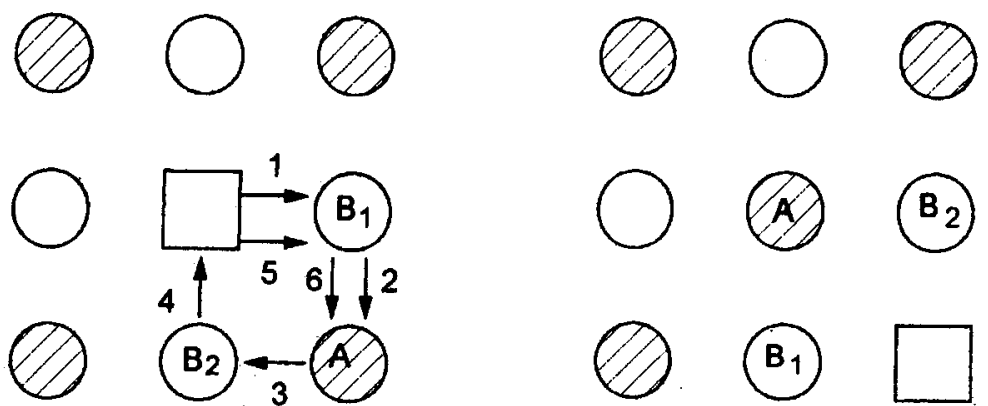

Fig. 1. A square planar analogue of the $6 \mathrm{JC}$ mechanism

Recently, the present authors showed that in the stoichiometric B2 structure with antistructural disorder the vacancy-wind factor $S$ for the (pure) $6 \mathrm{JC}$ is 0.42 [11]. This value is independent of the relative diffusion rates of the individual atomic species. Indeed, even if the diffusion rates of the atomic components are equal, $S$ still takes the value of 0.42 . This is a very unusual finding because it implies that even in the absence of a vacancy flux, i.e. when the two intrinsic diffusion coefficients are equal and there is no marker shift, there is still a non-trivial value for $S$. Note that Manning's result (Eqn 2) results in $\mathrm{S}$ being equal to unity in these circumstances. This interesting finding comes about because there are, in fact, two types of 6JC. In the first, the vacancy starts the $6 \mathrm{JC}$ from the $\alpha$-sublattice (home sublattice of the A atoms), this is the $\alpha-6 \mathrm{JC}$. In the second, the vacancy starts the $6 \mathrm{JC}$ from the $\beta$-sublattice (home sublattice of the $\mathrm{B}$ atoms), this is the $\beta-6 \mathrm{JC}$. During the operation of each type of $6 \mathrm{JC}$ there is a net vacancy flux producing a vacancy-wind effect, but these vacancy fluxes are in opposite directions. When the diffusivities are equal, the $\alpha-6 \mathrm{JC}$ and the $\beta-6 \mathrm{JC}$ also contribute equally to the overall 
diffusion. The result then is an overall net flux of vacancies of zero. However, the vacancy-wind effects resulting from the operation of each individual cycle do not cancel out. Computer simulations have verified this result and have shown a smooth gradation in $\mathrm{S}$ from very high temperatures, where the influence of the $6 \mathrm{JC}$ is negligible and $\mathrm{S}$ equals unity, to low temperatures where the $6 \mathrm{JC}$ dominates and $\mathrm{S}$ clearly converges to 0.42 [11]. Interestingly, the off-diagonal or cross phenomenological coefficient $\mathrm{L}_{\mathrm{AB}}$ is zero for both types of $6 \mathrm{JC}$, the result $\mathrm{S}=0.42$ actually comes from the diagonal coefficients $\mathrm{L}_{\mathrm{AA}}$ and $\mathrm{L}_{\mathrm{BB}}$.

These findings are confined to precisely the stoichiometric composition. Chemical diffusion is, of course, associated with compositional changes. One must then ask how relevant these findings really are to chemical diffusion in an intermetallic? In order to answer this it is necessary to extend the $6 \mathrm{JC}$ concept to include existing antistructural atoms, either introduced by nonstoichiometry (extrinsic defects), or those introduced by thermal activation (intrinsic defects). Many years ago, Domian and Aaronson [12] sketched a diffusion kinetics formalism built on the $6 \mathrm{JC}$ itself as the basic 'unit' of the diffusion process. The omission of correlation effects in that formalism meant that only a qualitative picture was possible at that time. Recently, the present authors proposed a detailed and comprehensive $6 \mathrm{JC}$ diffusion kinetics theory that includes correlation effects. The initial theory allowed only for the participation of antistructural atoms of one atomic species [13, 14]. This was later extended [15] to the general case where there is a composition and temperature dependent equilibrium between antistructural atoms of both species, see, for example, Chang and Neumann [16]. The excellent agreement seen between the results of this $6 \mathrm{JC}$ diffusion kinetics theory and computer simulation (where only single vacancy jumps were specified) indicates that the physics of the diffusion process at high levels of order can be well described by this new theory. In the present paper we analyse the implications of this theory on the behaviour of $S$ at different compositions and temperature and different energy asymmetries of the atomic species. Again we make use of computer simulation to test the theory and attempt to ascertain the limits of its use in the context of S.

\section{Theory}

It is well-known that $\mathrm{S}$ can be expressed exactly in terms of the phenomenological coefficients and the tracer diffusion coefficients, see for example, refs. $11,17,18$

$$
S=\frac{k T}{N}\left(\frac{c_{B}^{2} L_{A A}+c_{A}^{2} L_{B B}-2 c_{B} c_{A} L_{A B}}{c_{B} c_{A}\left(c_{A} D_{B^{*}}+c_{B} D_{A^{*}}\right)}\right)
$$


also ref. 20) and they also lead to displacements of tracer $A$ and $B$ atoms. Next, the ' $w_{2}$ jump' is a special truncated cycle consisting of two individual B jumps (one of which is the antistructural atom). This process has a much higher frequency than other $\alpha-6 \mathrm{JCs}$. This 'jump' only leads to displacements of tracer B atoms. Finally, in the Ising alloy model used here, the 'dissociation' and 'association' jumps, $w_{3}$ and $w_{4}$, are found to be identical to $\mathrm{w}_{0}$.

Physically, the process of diffusion in the presence of antistructural atoms of B in the Ising alloy model using, say, $\alpha-6 \mathrm{JC}$ can be described in the following way. There is no binding of the $\alpha-6$ JC vacancy to the antistructural $B$ atom $\left(w_{3}=w_{4}\right)$. The vacancy moves relatively slowly through the lattice, largely by isolated pure $\alpha-6 \mathrm{JCs}\left(\mathrm{w}_{0}\right)$. When the vacancy is close to an antistructural $B$ atom, the vacancy is engaged in either the faster rotational $\alpha-6 \mathrm{JCs}\left(\mathrm{w}_{1}\right)$ around the antistructural $\mathrm{B}$ atom or the even faster $\left(\mathrm{w}_{2}\right)$ jumps directly involving the $\mathrm{B}$ atom. This behaviour can be expressed as:

$$
\mathrm{w}_{2}>\mathrm{w}_{1}^{3}>\left(\mathrm{w}_{1}{ }^{2}+\mathrm{w}_{1}{ }^{1}\right) / 2>\mathrm{w}_{0}=\mathrm{w}_{3}=\mathrm{w}_{4},
$$

where the superscripts on the $\mathrm{w}_{1}$ jumps refer to the rotational jump sub-types mentioned above and described in detail $[13,14]$.

For excess B compositions Belova and Murch $[13,14]$ originally divided the $\alpha-6 \mathrm{JC}$ into two contributions, $\alpha-6 \mathrm{JC}, 1$ and $\alpha-6 \mathrm{JC}, 2$ corresponding to Regions 1 and 2 respectively, and analogously for the $\beta-6 \mathrm{JC}$. Region 1 refers to the region in the vicinity of an antistructural B atom and Region 2 refers to the remainder of the lattice. (In effect, in Region 2, diffusion is via pure 6JCs.) This was later generalized [15] by recognizing the co-existence of antistructural A and B atoms at all compositions and an equilibrium between them. Region 1 then refers to the region in the vicinity of an antistructural $B$ atom and an antistructural $\mathrm{A}$ atom. Region 2 refers to the remainder of the lattice where both the pure $\alpha-6 \mathrm{JC}$ and $\beta-6 \mathrm{JC}$ operate. There is also an assumption that the antistructural atoms do not interact with one another (this is in the spirit of the calculation of enhancement factors using the 5-frequency model [17]) and a further assumption that there are no cross-correlations in displacements a) between $\alpha-6 \mathrm{JCs}$ and $\beta-6 \mathrm{JCs}$ and b) between the two regions of a given type of $6 \mathrm{JC}$.

Belova and Murch [15] showed that the complete expressions for the tracer correlation factors for the $\mathrm{A}$ and $\mathrm{B}$ atoms are: 
where $\mathrm{N}$ is the number of sites per unit volume and $\mathrm{k}$ and $\mathrm{T}$ have their usual meanings. Eqn 4 can also be re-expressed in terms of the collective correlation factors (the correlated parts of the phenomenological coefficients $[18,19])$ and the tracer correlation factors:

$$
S=\frac{c_{B} f_{A A}+c_{A} g f_{B B}-2 c_{A} f_{A B}^{(A)}}{c_{B} f_{A}+c_{A} g f_{B}}
$$

where $f_{A A}, f_{B B}$ and $f_{A B}{ }^{(A)}$ are the collective correlation factors and $g$ is the ratio of number of $B$ jumps per atom to the number of $A$ jumps per atom. Thus, one way to calculate $S$ is to calculate individually the various types of correlation factors. This is done here.

For convenience, in the following we make use of the Ising alloy model where there are three pair interaction energies $\mathrm{E}_{\mathrm{AA}}, \mathrm{E}_{\mathrm{BB}}$ and $\mathrm{E}_{\mathrm{AB}}$ between $A A, B B$ and $A B$ pairs. We define $\Delta_{\mathrm{A}}=\mathrm{E}_{\mathrm{AA}}-\mathrm{E}_{\mathrm{AB}}, \Delta_{\mathrm{B}}=\mathrm{E}_{\mathrm{BB}}-\mathrm{E}_{\mathrm{AB}}$ and $\Delta=\Delta_{\mathrm{A}}-\Delta_{\mathrm{B}}$ and the ordering energy $\mathrm{E}$ is given by $\mathrm{E}=\mathrm{E}_{\mathrm{AA}}+\mathrm{E}_{\mathrm{BB}}-2 \mathrm{E}_{\mathrm{AB}}$ and asymmetry parameter $\mathrm{U}=\Delta / \mathrm{E}$. However, the formalism is not limited to this model, but can be adapted to others: the main requirement is a detailed set of migration and formation energies for the variants of the $6 \mathrm{JC}$ involving an existing antistructural atom. For example, an embedded atom method type of calculation could in principle provide this information [20].

At the level of the Bragg-Williams approximation it is easily shown that the sublattice occupations are given by the equilibrium equation [15-17]:

$$
\frac{c_{\Lambda}^{\beta} c_{B}^{\alpha}}{c_{A}^{\alpha} c_{B}^{\beta}}=\exp \left(-8\left(1-2 \delta^{\prime}\right) E / k T\right)
$$

The notation $c_{A}^{\beta}$ is used to represent the fraction of $A$ atoms on the $\beta$ sublattice and so on. For convenience, the parameter $\left.\delta^{\prime}=\left(c_{B}{ }^{\alpha}+c_{A}{ }^{\beta}\right) / 2\right)$ is used. The sublattice occupations themselves are related to each other and the overall composition of $B$ atoms $c_{B}$ by

$$
c_{A}^{\alpha}=1-c_{B}^{\alpha} ; c_{B}^{\beta}=2 c_{B}-c_{B}^{\alpha} ; c_{A}^{\beta}=1-2 c_{B}+c_{B}^{\alpha}
$$

Belova and Murch [13-15] adopted the nomenclature of the well-known 'five frequency model' to describe the various types of jump-units of the $6 \mathrm{JC}$. Consider, for example, the $\alpha-6$ JC (the prefix indicates the vacancy starts from the $\alpha$ sublattice). First, there is the ' $w_{0}$ jump', which is simply the pure $\alpha-6 \mathrm{JC}$ itself (this leads to displacements of tracer $A$ and $B$ atoms). Next, the ' $w_{1}$ jump', the rotational jump, is a set of four subtypes of the $\alpha-6 \mathrm{JC}$ that occur in roughly a rotational sense around an antistructural $\mathrm{B}$ atom. These $\alpha$-6JCs only involve the B atom indirectly via its energy of interaction (see 


$$
\begin{aligned}
\mathrm{f}_{\mathrm{A}} & =\frac{\exp \left\{\left(-7 \mathrm{c}_{\mathrm{A}}^{\beta} \Delta_{\mathrm{B}}+\mathrm{c}_{\mathrm{B}}^{\alpha} \Delta_{\mathrm{A}}-\mathrm{c}_{\mathrm{B}}^{\alpha} \mathrm{c}_{\mathrm{A}}^{\beta} \mathrm{E}\right) / \mathrm{kT}\right\}}{1-\mathrm{c}_{\mathrm{B}}^{\alpha}}\left[4\left(1-23 \delta^{\prime}\right)\left(\frac{\mathrm{w}_{0 \alpha} \mathrm{c}_{\mathrm{B}}^{\alpha}}{1-\mathrm{c}_{\mathrm{A}}^{\beta}} \exp \left\{8(1-2 \delta) \Delta_{\mathrm{B}} / \mathrm{kT}\right\}+2 \mathrm{w}_{0 \beta}\right)\right. \\
& \left.\left.+\mathrm{c}_{\mathrm{A}}^{\beta}\left[14 \mathrm{w}_{1 \beta}^{3}+25\left(\mathrm{w}_{1 \beta}^{1}+\mathrm{w}_{1 \beta}^{2}\right)\right]+\frac{24.1\left(\mathrm{c}_{\mathrm{B}}^{\alpha} / 2\right)^{2}}{1-\mathrm{c}_{\mathrm{A}}^{\beta}}\left[\mathrm{w}_{1 \alpha}^{3}+1.5\left(\mathrm{w}_{1 \alpha}^{1}+\mathrm{w}_{1 \alpha}^{2}\right)\right] \exp \left\{8(1-2 \delta) \Delta_{\mathrm{B}} / \mathrm{kT}\right\}\right]\right] \\
\mathrm{f}_{\mathrm{B}}= & \frac{\exp \left\{\left(-7 \mathrm{c}_{\mathrm{B}}^{\alpha} \Delta_{\mathrm{B}}+\mathrm{c}_{\mathrm{A}}^{\beta} \Delta_{\mathrm{A}}-\mathrm{c}_{\mathrm{B}}^{\alpha} \mathrm{c}_{\mathrm{A}}^{\beta} \mathrm{E}\right) / \mathrm{kT}\right\}}{1-\mathrm{c}_{\mathrm{A}}^{\beta}}\left[4\left(1-23 \delta^{\prime}\right)\left(\frac{\mathrm{w}_{0 \beta} \mathrm{c}_{\mathrm{A}}^{\beta}}{1-\mathrm{c}_{\mathrm{B}}^{\alpha}} \exp \left\{8(1-2 \delta) \Delta_{\mathrm{A}} / \mathrm{kT}\right\}+2 \mathrm{w}_{0 \alpha}\right)\right. \\
& \left.+\mathrm{c}_{\mathrm{B}}^{\alpha}\left[14 \mathrm{w}_{1 \alpha}^{3}+25\left(\mathrm{w}_{1 \alpha}^{1}+\mathrm{w}_{1 \alpha}^{2}\right)\right]+\frac{24.1\left(\mathrm{c}_{\mathrm{A}}^{\beta} / 2\right)^{2}}{1-\mathrm{c}_{\mathrm{B}}^{\alpha}}\left[\mathrm{w}_{1 \beta}^{3}+1.5\left(\mathrm{w}_{1 \beta}^{1}+\mathrm{w}_{1 \beta}^{2}\right)\right] \exp \left\{8(1-2 \delta) \Delta_{\mathrm{A}} / \mathrm{kT}\right\}\right]
\end{aligned}
$$

where $w_{0 \beta}$ is the frequency of a pure $\beta-6 \mathrm{JC}$ and so on.

$$
\begin{aligned}
& \mathrm{w}_{0 \beta}=v \exp \left\{\left(-6 \mathrm{E}\left(1-2 \delta^{\prime}\right)+7 \mathrm{c}_{\mathrm{A}}^{\beta} \Delta_{\mathrm{A}}+\mathrm{c}_{\mathrm{B}}^{\alpha} \Delta_{\mathrm{B}}\right) / \mathrm{kT}\right\}, \\
& \mathrm{w}_{0 \alpha}=v \exp \left\{\left(-6 \mathrm{E}\left(1-2 \delta^{\prime}\right)+7 \mathrm{c}_{\mathrm{B}}^{\alpha} \Delta_{\mathrm{B}}+\mathrm{c}_{\mathrm{A}}^{\beta} \Delta_{\mathrm{A}}\right) / \mathrm{kT}\right\}, \\
& \mathrm{w}_{1 \beta}^{3}=v \exp \left\{\left(-5 \mathrm{E}\left(1-2 \delta^{\prime}-\mathrm{c}_{\mathrm{B}}^{\alpha}\right)+\Delta_{\mathrm{A}}+6 \mathrm{c}_{\mathrm{A}}^{\beta} \Delta_{\mathrm{A}}+\mathrm{c}_{\mathrm{B}}^{\alpha} \Delta_{\mathrm{B}}\right) / \mathrm{kT}\right\}, \\
& \mathrm{w}_{1 \alpha}^{3}=v \exp \left\{\left(-5 \mathrm{E}\left(1-2 \delta^{\prime}-\mathrm{c}_{\mathrm{A}}^{\beta}\right)+\Delta_{\mathrm{B}}+6 \mathrm{c}_{\mathrm{B}}^{\alpha} \Delta_{\mathrm{B}}+\mathrm{c}_{\mathrm{A}}^{\beta} \Delta_{\mathrm{A}}\right) / \mathrm{kT}\right\}, \\
& \mathrm{w}_{1 \beta}^{2}=v \exp \left\{\left(-5 \mathrm{E}\left(1-2 \delta^{\prime}-\mathrm{c}_{\mathrm{B}}^{\alpha}\right)+7 \mathrm{c}_{\mathrm{A}}^{\beta} \Delta_{\mathrm{A}}+\mathrm{c}_{\mathrm{B}}^{\alpha} \Delta_{\mathrm{B}}\right) / \mathrm{kT}\right\}, \\
& \mathrm{w}_{1 \alpha}^{2}=v \exp \left\{\left(-5 \mathrm{E}\left(1-2 \delta^{\prime}-\mathrm{c}_{\mathrm{A}}^{\beta}\right)+7 \mathrm{c}_{\mathrm{B}}^{\alpha} \Delta_{\mathrm{B}}+\mathrm{c}_{\mathrm{A}}^{\beta} \Delta_{\mathrm{A}}\right) / \mathrm{kT}\right\}, \\
& \mathrm{w}_{1 \beta}^{1}=v \exp \left\{\left(-6 \mathrm{E}\left(1-2 \delta^{\prime}+\mathrm{c}_{\mathrm{A}}^{\beta}\right)+\Delta_{\mathrm{A}}+7 \mathrm{c}_{\mathrm{A}}^{\beta} \Delta_{\mathrm{A}}+2 \delta^{\prime} \Delta_{\mathrm{B}}\right) / \mathrm{kT}\right\}, \\
& \mathrm{w}_{1 \alpha}^{1}=v \exp \left\{\left(-6 \mathrm{E}\left(1-2 \delta^{\prime}+\mathrm{c}_{\mathrm{B}}^{\alpha}\right)+\Delta_{\mathrm{B}}+7 \mathrm{c}_{\mathrm{B}}^{\alpha} \Delta_{\mathrm{B}}+\delta^{\prime} \Delta_{\mathrm{A}}\right) / \mathrm{kT}\right\},
\end{aligned}
$$

where $v$ is the attempt frequency. (For simplicity in the present analysis, all the attempt frequencies for the various jumps and the two species are put equal.)

Belova and Murch [15] showed that the collective correlation factors are given by:

$$
\begin{aligned}
\mathrm{f}_{\mathrm{AA}} & =\frac{\mathrm{c}_{\mathrm{B}}^{\alpha} \exp \left\{\left(8 \Delta_{\mathrm{B}}-7 \mathrm{c}_{\mathrm{B}}^{\alpha} \Delta_{\mathrm{B}}\right) / \mathrm{kT}\right\}}{\left(1-\mathrm{c}_{\mathrm{B}}^{\alpha}\right)\left(1-\mathrm{c}_{\mathrm{A}}^{\beta}\right)}\left[2 \mathrm{c}_{\mathrm{B}}^{\alpha}\left(2 \mathrm{w}_{1 \alpha}^{3}+3\left(\mathrm{w}_{1 \alpha}^{1}+\mathrm{w}_{1 \alpha}^{2}\right)\right)\right] \\
& +\frac{2 \mathrm{c}_{\mathrm{A}}^{\beta} \exp \left\{\left(\mathrm{c}_{\mathrm{B}}^{\alpha} \Delta_{\mathrm{B}}-7 \mathrm{c}_{\mathrm{A}}^{\beta} \Delta_{\mathrm{A}}\right) / \mathrm{k} T\right\}}{\left(1-\mathrm{c}_{\mathrm{B}}^{\alpha}\right)}\left[\mathrm{w}_{1 \beta}^{3}+4.3\left(\mathrm{w}_{1 \beta}^{1}+\mathrm{w}_{1 \beta}^{2}\right)\right] \\
& +5(1-23 \delta) \mathrm{w}_{0 \alpha} \exp \left\{\left(-8 \Delta_{\mathrm{A}}+\mathrm{c}_{\mathrm{A}}^{\beta} \Delta_{\mathrm{A}}+\mathrm{c}_{\mathrm{B}}^{\alpha} \Delta_{\mathrm{B}}+8 \mathrm{c}_{\mathrm{B}}^{\alpha} \Delta_{\mathrm{A}}\right) / \mathrm{k} T\right\} / \mathrm{c}_{\mathrm{A}}^{\beta},
\end{aligned}
$$




$$
\begin{gathered}
\mathrm{f}_{\mathrm{BB}}=\frac{\mathrm{c}_{\mathrm{A}}^{\beta} \exp \left\{\left(8 \Delta_{\mathrm{A}}-7 \mathrm{c}_{\mathrm{A}}^{\beta} \Delta_{\mathrm{A}}\right) / \mathrm{kT}\right\}}{\left(1-\mathrm{c}_{\mathrm{B}}^{\alpha}\right)\left(1-\mathrm{c}_{\mathrm{A}}^{\beta}\right)}\left[2 \mathrm{c}_{\mathrm{A}}^{\beta}\left(2 \mathrm{w}_{1 \beta}^{3}+3\left(\mathrm{w}_{1 \beta}^{1}+\mathrm{w}_{1 \beta}^{2}\right)\right)\right] \\
+\frac{2 \mathrm{c}_{\mathrm{B}}^{\alpha} \exp \left\{\left(\mathrm{c}_{\mathrm{A}}^{\beta} \Delta_{\mathrm{A}}-7 \mathrm{c}_{\mathrm{B}}^{\alpha} \Delta_{\mathrm{B}}\right) / \mathrm{kT}\right\}}{\left(1-\mathrm{c}_{\mathrm{A}}^{\beta}\right)}\left[\mathrm{w}_{1 \alpha}^{3}+4.3\left(\mathrm{w}_{\mathrm{l} \alpha}^{1}+\mathrm{w}_{\mathrm{l \alpha}}^{2}\right)\right] \\
+5(1-23 \delta) \mathrm{w}_{0 \beta} \exp \left\{\left(-8 \Delta_{\mathrm{B}}+\mathrm{c}_{\mathrm{A}}^{\beta} \Delta_{\mathrm{A}}+\mathrm{c}_{\mathrm{B}}^{\alpha} \Delta_{\mathrm{B}}+8 \mathrm{c}_{\mathrm{A}}^{\beta} \Delta_{\mathrm{B}}\right) / \mathrm{k} T\right\} / \mathrm{c}_{\mathrm{B}}^{\alpha}, \\
\mathrm{f}_{\mathrm{AB}}^{(\mathrm{A})}=-\frac{2\left(\mathrm{c}_{\mathrm{B}}^{\alpha}\right)^{2} \exp \left\{\left(8 \Delta_{\mathrm{B}}\left(1-2 \delta^{\prime}\right)+\mathrm{c}_{\mathrm{B}}^{\alpha} \Delta_{\mathrm{B}}-7 \mathrm{c}_{\mathrm{A}}^{\beta} \Delta_{\mathrm{A}}\right) / \mathrm{kT}\right\}}{\left(1-\mathrm{c}_{\mathrm{B}}^{\alpha}\right)\left(1-\mathrm{c}_{\mathrm{A}}^{\beta}\right)}\left[2 \mathrm{w}_{1 \alpha}^{3}+3\left(\mathrm{w}_{1 \alpha}^{1}+\mathrm{w}_{1 \alpha}^{2}\right)\right] \\
-\frac{2 \mathrm{c}_{\mathrm{A}}^{\beta} \exp \left\{\left(\mathrm{c}_{\mathrm{B}}^{\alpha} \Delta_{\mathrm{B}}-7 \mathrm{c}_{\mathrm{A}}^{\beta} \Delta_{\mathrm{A}}-\mathrm{c}_{\mathrm{B}}^{\alpha} \mathrm{c}_{\mathrm{A}}^{\beta} \mathrm{E}\right) / \mathrm{kT}\right\}}{\left(1-\mathrm{c}_{\mathrm{B}}^{\alpha}\right)}\left[2 \mathrm{w}_{1 \beta}^{3}+3\left(\mathrm{w}_{1 \beta}^{1}+\mathrm{w}_{1 \beta}^{2}\right)\right], \\
\mathrm{f}_{\mathrm{AB}}^{(\mathrm{B})}=-\frac{2\left(\mathrm{c}_{\mathrm{A}}^{\beta}\right)^{2} \exp \left\{\left(8 \Delta_{\mathrm{A}}\left(1-2 \delta^{\prime}\right)+\mathrm{c}_{\mathrm{A}}^{\beta} \Delta_{\mathrm{A}}-7 \mathrm{c}_{\mathrm{B}}^{\alpha} \Delta_{\mathrm{B}}\right) / \mathrm{kT}\right\}}{\left(1-\mathrm{c}_{\mathrm{B}}^{\alpha}\right)\left(1-\mathrm{c}_{\mathrm{A}}^{\beta}\right)}\left[2 \mathrm{w}_{1 \beta}^{3}+3\left(\mathrm{w}_{1 \beta}^{1}+\mathrm{w}_{1 \beta}^{2}\right)\right] \\
-\frac{2 \mathrm{c}_{\mathrm{B}}^{\alpha} \exp \left\{\left(\mathrm{c}_{\mathrm{A}}^{\beta} \Delta_{\mathrm{A}}-7 \mathrm{c}_{\mathrm{B}}^{\alpha} \Delta_{\mathrm{B}}-\mathrm{c}_{\mathrm{B}}^{\alpha} \mathrm{c}_{\mathrm{A}}^{\beta} \mathrm{E}\right) / \mathrm{kT}\right\}}{\left(1-\mathrm{c}_{\mathrm{A}}^{\beta}\right)}\left[2 \mathrm{w}_{1 \alpha}^{3}+3\left(\mathrm{w}_{1 \alpha}^{1}+\mathrm{w}_{1 \alpha}^{2}\right)\right] .
\end{gathered}
$$

Finally, the ratio of numbers of B jumps to A jumps, g, is given by:

$$
g=\frac{c_{A} c_{B}^{\alpha}}{c_{B} c_{A}^{\alpha}} \exp \left\{8\left(\Delta_{B}-c_{\Lambda}^{\beta} E\right) / k T\right\}
$$

With these expressions in hand it is now possible to assemble $\mathrm{S}$ for the 6JC diffusion kinetics theory from Eqn 5, using Eqns 11-16 for the correlation factors along with the numerical solution of Eqn 6 .

Monte Carlo computer simulation of the tracer and collective correlation factors and $\mathrm{g}$ was performed using methods that have been described many times, see, for example, refs. 19,21. Typical computational conditions are given in ref. 15. The simulations were based entirely around single vacancy jumps: no $6 \mathrm{JC}$ information was provided to the simulation. S was calculated from Eqn 5.

\section{Results and Discussion}

In Fig 2 we present analytical (lower set of curves) and computer simulation results (symbols) for the vacancy wind factor $S$ as a function of composition $c_{A}$ at the temperatures: $\mathrm{T} / \mathrm{T}_{\mathrm{c}}=0.39,0.47,0.55,0.63,0.71\left(\mathrm{E} / \mathrm{kT}_{\mathrm{c}}=0.63\right.$ for this lattice $)$ and with the asymmetry parameter, $\mathrm{U}=0$. In the same figure we also include Manning's result (Eqn 3). 


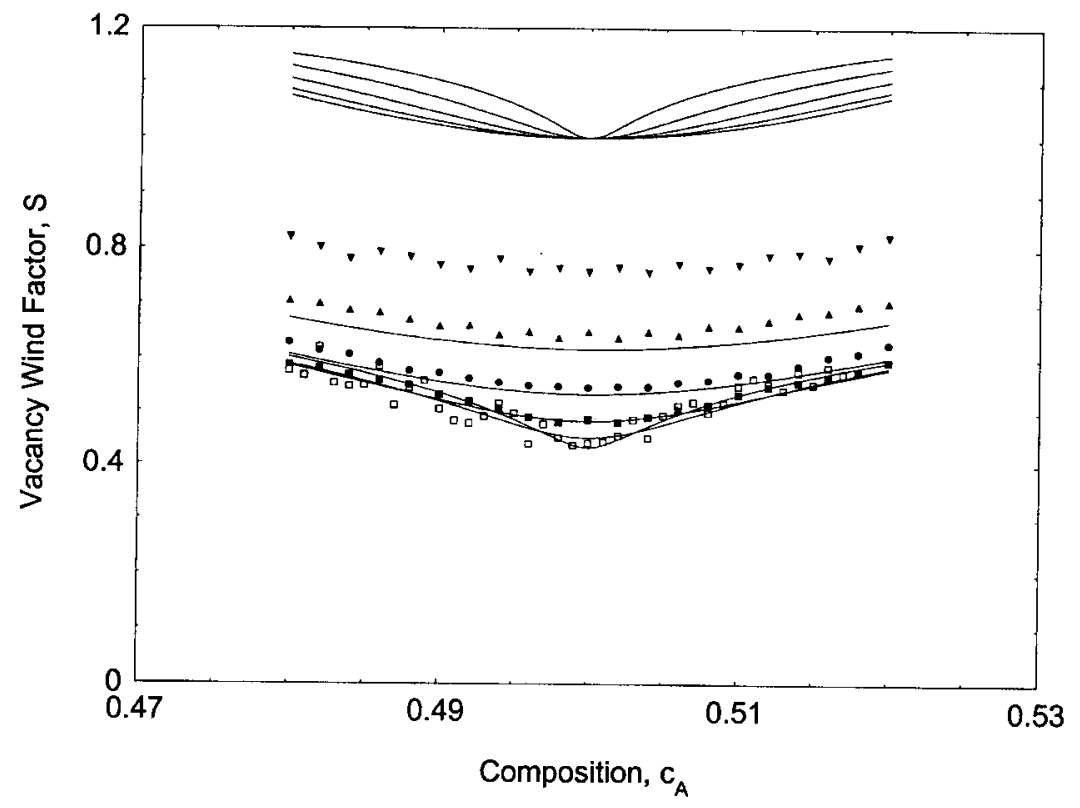

Fig. 2. The vacancy-wind factor $S$ as a function of composition $c_{A}$ with $U=0.0$ and at various values of $T / T_{c}$. Upper curves: analytical $6 \mathrm{JC}$ results using Manning's Eqn. 3; lower curves: analytical $6 \mathrm{JC}$ results using Eqn 5; data points: Monte Carlo results ( $\square: 0.39, \mathbf{\square}: 0.47, \bullet: 0.55, \mathbf{\Delta}: 0.63, \boldsymbol{\nabla}: 0.71$ ).

This is the upper set of curves. It is clear that at the lowest temperatures there is excellent agreement between the computer simulations (which are based around single vacancy jumps) and the analytical results (which are based around 6JC 'jumps'). It is noted that the lowest value of $\mathrm{S}$ occurs at the stoichiometric composition where it is approximately equal to 0.42 as predicted analytically [11]. As the temperature increases the value of $S$ increases towards unity. Discrepancies between simulations and theory do become apparent at the higher temperatures. This is expected since diffusion in the ordered structure will lose its $6 \mathrm{JC}$ character as disorder increases and other jump processes effectively corrupt the $6 \mathrm{JC}$ sequences. It can also be seen that the Manning results vary very little with temperature and remain at or above unity as expected. Note the Manning result $(S=1.0)$ at the stoichiometric composition for this symmetric $(U=0.0)$ case.

Real intermetallic B2 compounds with antistructural disorder typically have asymmetry parameters $U$ taking values of the order of 0.5 , see, for example, ref. 23 . Large values of $U$ (e.g. greater than about unity) will almost certainly be accompanied by structural vacancies. This goes outside the possibilities of the present $6 \mathrm{JC}$ formalism of course. It is appropriate then to explore $S$ for several cases of $U \neq 0.0$. One effect of $U \neq 0.0$ 


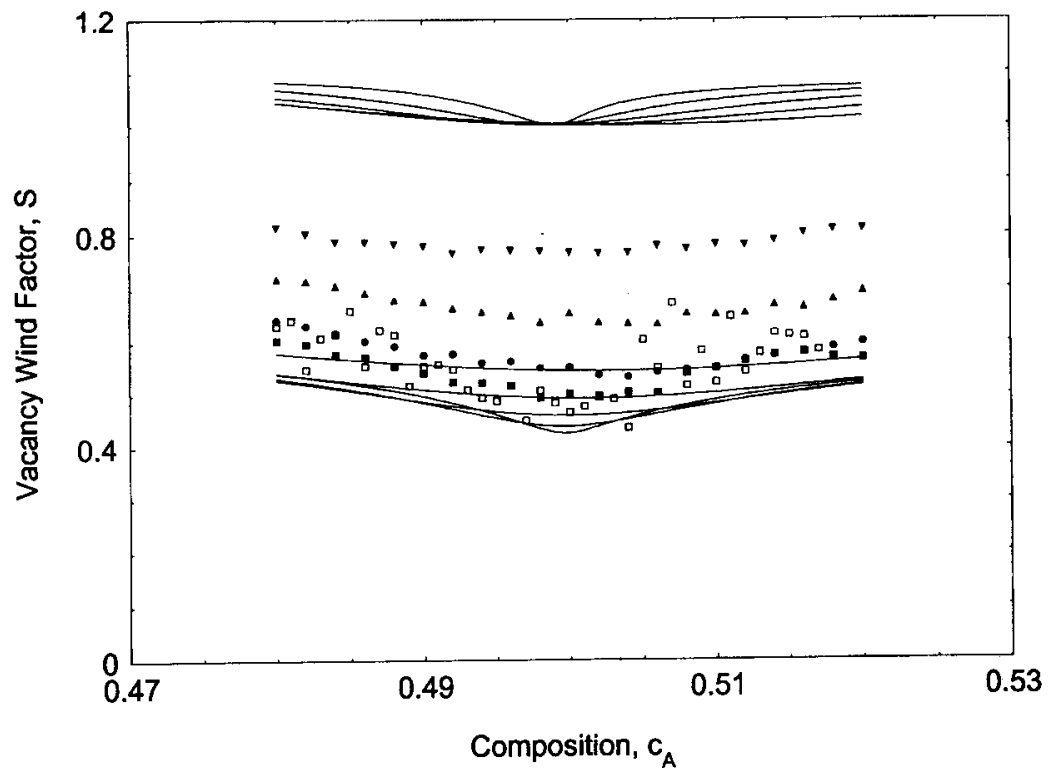

Fig. 3. The vacancy-wind factor $S$ as a function of composition $c_{A}$ with $U=0.125$ and at various values of $\mathrm{T} / \mathrm{T}_{\mathrm{c}}$. Upper curves: analytical $6 \mathrm{JC}$ results using Manning's Eqn. 3; lower curves: analytical $6 \mathrm{JC}$ results using Eqn. 5; data points: Monte Carlo results ( $\square: 0.39, \mathbf{=}: 0.47, \bullet: 0.55, \mathbf{\Delta}: 0.63, \nabla: 0.71)$.

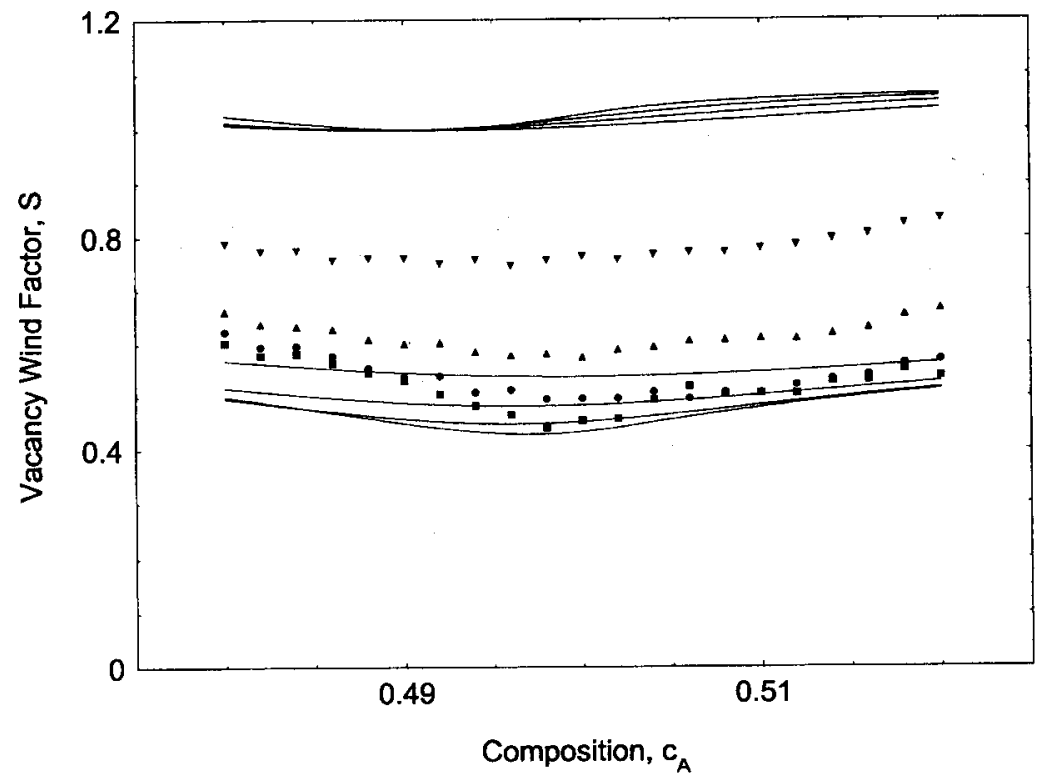

Fig. 4. The vacancy-wind factor $S$ as a function of composition $c_{A}$ with $U=0.5$ and at various values of $T / T_{c}$. Upper curves: analytical $6 \mathrm{JC}$ results using Manning's Eqn. 3; lower curves: analytical $6 \mathrm{JC}$ results using Eqn. 5; data points: Monte Carlo results ( $\mathbf{\square}: 0.47, \bullet: 0.55, \mathbf{\Delta}: 0.63, \mathbf{\nabla}: 0.71)$. 


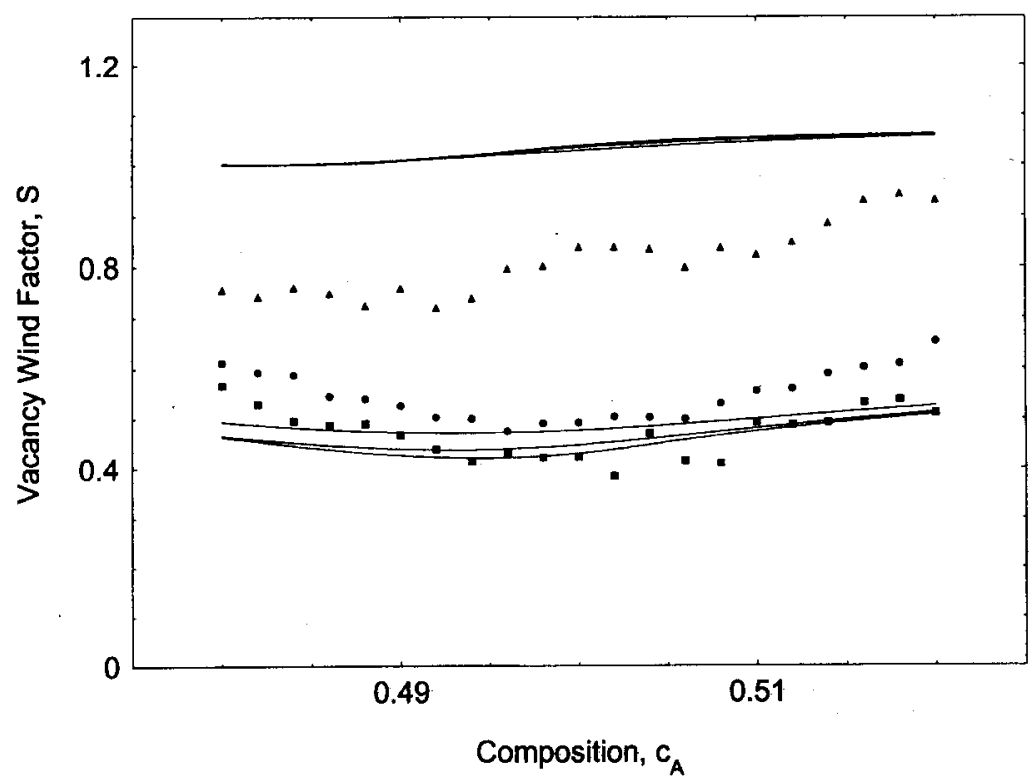

Fig. 5. The vacancy-wind factor $S$ as a function of composition $c_{A}$ with $U=0.875$ and at various values of $T / T_{c}$. Upper curves: analytical $6 \mathrm{JC}$ results using Manning's Eqn. 3; lower curves: analytical $6 \mathrm{JC}$ results using Eqn. 5; data points: Monte Carlo results (匹: $0.47, \bullet: 0.55, \triangle$ : 0.63 ).

is to alter the partitioning of the vacancies between the two sublattices [13-15]. At the stoichiometric composition, for example, this make one type of $6 \mathrm{JC}$ dominant over the other. $U \neq 0.0$ also changes the relative magnitudes of the various 6JC 'jumps'. In Figs. 3-5 we present further results for $S$ at values of $U=0.125,0.5$, and 0.875 . Although there is somewhat more scatter at the low temperatures the general behaviour remains much the same as above in Fig. 2.

\section{Conclusions}

It is clear that the analytical formalism based around the generalized $6 \mathrm{JC}$ mechanism describes the vacancy wind factor very well for the low temperatures/high levels of order where this mechanism is a major contributor to the diffusion process. From a practical point of view (without performing a full analysis and providing that the level of order is high), it would be reasonable as a first (rough) approximation to assume that $S \approx 0.5$ rather than to assume $\mathrm{S}=1.0$ as is often the case. The level of order itself can be established via knowledge of the long range order parameter, order/disorder temperature or thermodynamic activity, see for example ref. 17 . 


\section{Acknowledgments}

We wish to thank the Australian Research Council (Large Grants and Discovery Project Grants Schemes) for its support of this research. One of us (IVB) also wishes to thank the Australian Research Council for the award of a Queen Elizabeth II Fellowship.

\section{REFERENCES}

1. J.R. Manning, Diffusion Kinetics for Atoms in Crystals (Princeton, New Jersey: Van Nostrand-Reinhold), (1968).

2. J.R. Manning, Phys. Rev. B, 4, 1111 (1971).

3. I.V. Belova and G.E. Murch, Phil. Mag. A, 75, 1715 (1997).

4. G.E. Murch, Phil. Mag. A, 46, 575 (1982).

5. I.V. Belova and G.E. Murch, J. Phys. Chem. Solids, 60, 2023 (1999).

6. I.V. Belova and G.E. Murch, Phil. Mag. A, 78, 1085 (1998).

7. H.B. Huntington, N.C. Miller and V. Nersis, Acta Met., 9, 749 (1961).

8. E.W. Elcock and C.W. McCombie, Phys. Rev., 109, 605 (1958).

9. I.V. Belova, M.E. Ivory and G.E. Murch, Phil. Mag. A, 72, 871 (1995).

10. I.V. Belova and G.E. Murch, Phil. Mag. A, 80, 1481 (2000).

11. I.V. Belova and G.E. Murch, Phil. Mag. A, 81, 83 (2001).

12. H.A. Domian and H.I. Aaronson, Trans TMS-AIME, 230, 44 (1964).

13. I.V. Belova and G.E. Murch, Phil. Mag. A, 82, 269 (2002).

14. I.V. Belova and G.E. Murch, Phil. Mag. A, 82, 285 (2002).

15. I.V. Belova and G.E. Murch, J. Phys.: Condensed Matter, accepted for publication and in press.

16. Y.A. Chang and J.P. Neumann, Prog. Solid St. Chem., 14, 221 (1982).

17. A.R. Allnatt and A.B. Lidiard, Atom Transport in Solids, Cambridge University Press, (1993).

18. G.E. Murch, Phase Transformations in Materials, Chapter 2, edited by G. Kostorz, Wiley-VCH (2001).

19. A.R. Allnatt and E.L. Allnatt, Phil. Mag. A, 49, 625 (1984).

20. Y.Mishin and D. Farkas, Scripta Mat., 39, 625 (1998).

21. M. Athènes, P. Bellon and G. Martin, Phil. Mag. A, 76, 965 (1997).

22. G.E. Murch, Diffusion in Crystalline Solids, Chapter 7, edited by G.E.Murch and A.S.Nowick (Orlando, Florida: Academic Press), (1984).

23. I.V.Belova and G.E.Murch, Acta Mater., 46, 849 (1998). 
Defect and Diffusion in Metals

doi:10.4028/3-908450-75-6

The Six-Jump Cycle Diffusion Mechanism in Non-Stoichiometric B2 Intermetallics:

the Vacancy-Wind Factor

doi:10.4028/3-908450-75-6.95 